\title{
La función democrática de la arquitectura
}

\section{The democratic function of architecture}

\author{
Prof. Juan R. Castillo Molina, Dr. Eng. \\ Coordinador de Innovación Arquitectónica \\ Universidad Nacional Pedro Henríquez Ureña (UNPHU) \\ Santo Domingo, República Dominicana \\ jrcastillo@unphu.edu.do = https://orcid.org/0000-0001-5975-4307
}

Fecha de recepción: 5 de octubre de 2020

Fecha de aceptación: 3 de noviembre de 2020

Fecha de publicación: 1 de enero de 2021

Favor citar este artículo de la siguiente forma:

Castillo Molina, J. (2021). La función democrática de la arquitectura.

AULA Revista de Humanidades y Ciencias Sociales, 8-16

https: //doi.org/10.33413/aulahcs.2021.67i1.148

\section{RESUMEN}

El flujo de conocimiento de una industria basada en riesgos (aeroespacial) hacia otra encargada de producir objetos habitables terrestres, es necesario para ilustrar la función democrática de la arquitectura-En ese orden, el objetivo de este artículo es determinar cómo esa dinámica ilustra la acción liberadora de la profesión, usando una metodología de análisis que toma los conceptos autenticidad, policromía, exactitud, responsividad y habitabilidad (estrategias seminales aeroespaciales); aplicados a la planificación arquitectónica. Como resultado, se descubren principios originarios, componentes intangibles (informadores) y tangibles (formadores) que hacen de la arquitectura un campo eficaz para expandir libertad intelectual.

Palabras clave: aeroespacial, hábitat, sistema, transferencia de conocimiento, unicidad.

\begin{abstract}
The source of knowledge from an industry based on risks (aerospace) to another leading the production of habitable terrestrial places is necessary to illustrate the democratic function of architecture. Consequently, the purpose of this article is to determine how that dynamic is the liberating action of the profession through analyzing the concepts of Authenticity, Polychromy, Precision, Responsivity and Habitability (seminal aerospace strategies) applied to architectural planning. As a result, it was discovered the Originary Principles, intangible elements (information) and tangible ones (shapers) that establish architecture as one of the fields able to expand intellectual freedom.
\end{abstract}

Keywords: citizenship, aerospace, habitat, knowledge transfer, system, unicity. 


\section{Introducción}

La interdisciplinariedad expone la función democrática de la arquitectura apoyándose en ideas que puedan definir nuevas formas de habitar el planeta. Usar las máquinas como fuente de inspiración es una de esas ideas heredadas del pasado milenio que continúan informando a aquellas profesiones que moldean el ambiente construido (McCarter, 1987). Las máquinas fueron las bases de dos tendencias, una que la usó como patrón estético y otra que la tomó como una analogía de progreso científico -lo utilitario. En este artículo, ambas opciones se dirigen hacia la formulación de un concepto democrático basado en intercambios profesionales, donde la arquitectura se utilice para producir estructuras que inspiren el próximo nivel de civilización. Tradicionalmente, las ingenierías se inclinan hacia infraestructuras, mientras la arquitectura se enfoca en lo plástico, y es ventajoso alejarse de esa confrontación para llegar a reflexiones profesionales avanzadas. En el presente ensayo, la democracia equivale a la libertad de utilizar conocimientos foráneos a nuestra profesión, para responder a los retos impuestos por el presente milenio.

Históricamente, arquitectura e ingeniería habían trabajado al unísono, pero algo sucedió en el paso hacia la presente época que evolucionaron como campos separados descubriendo avances aisladamente (Cole, 2006). Hoy, existen procesos y plataformas digitales que conectan todos los aspectos de ambas profesiones en una plataforma única, dando oportunidad a intercambios disciplinares exitosos (Casey \& McWilliams, 2010); aunque por razones de espacio es imposible agotar ese aspecto en este artículo, sí se determinará cómo la interdisciplinariedad es la función democrática de la arquitectura, tomando los conceptos autenticidad, policromía, exactitud, responsividad y habitabilidad del mundo aeroespacial, y aplicándolos a la ideación arquitectónica. Como resultado, se descubren principios originarios, componentes intangibles (informadores) y tangibles (formadores) que hacen de la arquitectura un campo capaz de expandir libertad intelectual a través de la disciplinariedad cruzada.

\section{Antecedentes}

Lo aeroespacial es una industria que abarca todos los procesos humanos de transformación desde el psicológico hasta la fabricación del confort en zonas extremas. Sus productos consisten en toda estructura o nave que se instale o se traslade fuera de la atmósfera terrestre (Asimov, 1974). Conquistar el espacio aéreo es antiguo, pues volar artificialmente está en los bajorrelieves del hombre-pájaro esculpidos en las piedras de la Isla de Pascua, en el trabajo teórico de Leonardo da Vinci, en los vuelos experimentales en globo aerostático del siglo 18, en el logro de los hermanos Wright y en las ciudades flotantes propuestas por Krutikov en 1928 (Magomedov, 2015).

La arquitectura, como profesión renacentista, insiste en la reinvención constante de ambientes artificiales utilizando recursos plásticos - medios de expresión tangibles. Históricamente, una de sus fuentes es la industria armamentista de la cual extrae soluciones para materiales, formas $\mathrm{y}$ herramientas que eventualmente fueron beneficiosas, como la computadora, por ejemplo. A mediados del siglo pasado la profesión se inspiró en lo cósmico siguiendo el afán de la carrera espacial y su plan de alcanzar nuevas fronteras universales. Grupos como Archigram aprovechan el tema para crear lugares que alojen los nuevos estilos de vida que las revoluciones sociales de la época introdujeron. Los Metabolistas japoneses, aunque inspirados en temas biológicos, también utilizaron todo conocimiento aeroespacial a disposición. Las oportunidades de experimentación de la nueva tendencia sucedieron en las ferias internacionales, especialmente la Expo Osaka 1970 (Ota, 2011). Recientemente, la estación científica Halley VI en la Antártica es un ejemplo de implementación de materiales aeroespaciales, resultando en una arquitectura que demuestra la efectividad del 
uso democrático del conocimiento cruzado (Castillo Molina, 2019).

\section{Estrategias aeroespaciales}

En el presente artículo, la interdisciplinariedad arquitectónica está basada en cinco estrategias deducidas de lo aeroespacial: autenticidad, policromía, exactitud, responsividad y habitabilidad. Todas están enfocadas en la manufactura, fabricación y desempeño de objetos vitales para el confort y la seguridad humana, aspectos necesarios para planificar arquitectura.

\section{Autenticidad}

La autenticidad está representada por la huella industrial dejada en los elementos principales del conjunto. Es la característica visual del todo, y es el rasgo distintivo de cómo cada parte fue manufacturada. Sus distinciones se deben a las especificaciones y manejo del material en el proceso, lo que evita que algo que haya pasado por un proceso de fabricación genuino pueda ser balanizado fácilmente; podría falsificarse, pero sería solamente una apariencia y quedaría reducido a réplicas.

La autenticidad es una cualidad antigua. Se encuentra en el concreto cuando ha sido desencofrado y dejado sin recubrimiento. También sucede cuando los elementos que debieron ser efímeros en el proceso constructivo fueron dejados junto a la estructura y terminaron formando parte del resultado exterior final, como es el caso del techo de las chozas Obus, de la tribu Musgum en Cameron, que fueron hechas con pilares en hileras acercadas, con protuberancias para ir colocando los pies mientras se sube hasta la linterna de la cúpula. Al terminar, los pilares quedan embebidos en la terracota definiendo la textura tridimensional que las caracteriza exteriormente (Chin, 2010).

En lo aeroespacial, la autenticidad está evidenciada por las esquinas en ángulo curvo, que resultan cuando el metal es sometido a presión entre una prensa y un molde. Es difícil conseguir aristas filosas con los metales, pues la naturaleza del material hace que los mismos se plieguen curveados, al menos que se corten las piezas por separado y sean soldadas posteriormente. En la década de 1960, el ángulo curvo fue temático, se transfirió del metal al concreto y luego al plástico. Todo se unía en ángulo curvo (pisos, paredes, esquinas y aristas de ventanas) indistintamente de lo que fueran hechos, concluyendo en productos que presagiaban un estilo «espacial» para el siglo 21 basado en soft edges (Garner, 1996).

\section{Policromía}

Policromía resulta al codificar objetos de servicio por color indicando el propósito o peligro de los mismos. No todas las partes se pigmentan siguiendo un código, solo aquellas que son parte del sistema de soporte vital o de emergencia, es decir, la ventilación, calefacción, electricidad, data, bordes peligrosos y otras que aseguren el buen funcionamiento y seguridad.

En la industria aeroespacial todo lo vital debe ser indicado y resaltado para guiar a los usuarios, en momentos de emergencia especialmente. La aeronáutica y las industrias pesadas, donde se manejan y fabrican elementos que podrían poner en peligro la vida humana, usan códigos de colores también. Igualmente, la policromía ha sido tradición en arquitectura e ingeniería, ya que en los planos y en la ejecución de los sistemas hidráulicos de los edificios aplican la coloración a los diversos elementos, y en los gráficos tridimensionales de los análisis estructurales se necesita para diferenciar cada elemento analizado en simulación digital, por ejemplo (Sasaki, 2005).

Una alternativa a la policromía en la arquitectura es codificar sus elementos por diagrama de barras digitales u otro recurso no visual, pero se necesitan intermediarios como aparatos lectores o un proceso de verificación de data. Sin embargo, el color se identifica casi instantáneamente, garantizando el discernimiento de las partes de un sistema arquitectónico, algo útil para tener una advertencia inmediata en caso de 
emergencia. La policromía se recomienda en arquitectura para las partes vitales de una estructura, aun cuando estén ocultas detrás de cierres o compuertas que puedan eventualmente abrirse para mantenimiento o sustitución.

\section{Exactitud}

Un objeto es exacto cuando alcanza precisión en la formulación, manufactura y fabricación, $\mathrm{y}$ ha pasado por un proceso de verificación y validación humano. La exactitud se logra al eliminar todo error que pueda comprometer el propósito de cada elemento, y se muestra como rasgo distintivo en la apariencia del conjunto al evidenciar el cuidado con el que fue pensada, fabricada y ensamblada cada parte del todo (Oxman \& Oxman, 2010).

En lo aeroespacial, los elementos que han pasado por un proceso de perfeccionamiento se distinguen por su moderación y austeridad, evitando excesos. La exactitud da la oportunidad de ir más allá de la conveniencia, pues como goce estético expresa la satisfacción de llegar a la perfección. Lo exacto exige que las piezas y el conjunto respondan al mínimo uso de materias primas, que el espesor de cada material y componente sea reducido a lo imprescindible, garantizando la reducción del peso y la optimización de energía, ambos aspectos vitales para el éxito de una implementación consciente medioambientalmente. La arquitectura existe en lugares naturales o fabricados ocupando un espacio preciso, por lo tanto, es imperativo que sea exacta en el tamaño de sus partes para lograr un manejo moderado de materiales y para disminuir el inevitable daño colateral que un hábitat artificial produce en los sitios a pesar del cuidado que se tenga al implementarlos.

\section{Responsividad}

Responsividad sucede cuando partes específicas de un objeto responden automáticamente a una condición externa usando sensores. En la industria aeroespacial, la responsividad es crucial para lidiar con los cambios de temperatura y presión, niveles de radiación, los acoplamientos entre naves, el manejo de las compuertas y las patas de aterrizaje. La red de sensores es necesaria para mediar entre la necesidad humana y la indumentaria aeroespacial, y para garantizar una experiencia fluida entre los aparatos y el usuario. El control de la producción y el uso de energía son procesos automáticos en los hábitats y naves espaciales gracias a la ubicuidad de la computación. Estos procesos hacen que todo lugar automatizado sea un ambiente controlable y seguro para el desarrollo de actividades humanas (Dunn, 2012). La responsividad es también un sistema de alerta que, en caso de bloquearse, puede finalmente manipularse análogamente -manualmente.

La responsividad es programada. El cálculo y la precisión automáticos ante cambios externos a los hábitats, es posible debido a la computación y recientemente a las exploraciones e implementaciones de la cibernética. Los ambientes artificiales como la arquitectura deben ser adaptables a las condiciones atmosféricas y geológicas del sitio a colocarse, y su éxito dependerá de cuán automáticamente respondan a las eventualidades del lugar y al uso humano.

\section{Habitabilidad}

Habitabilidad sucede cuando la atmósfera artificial de un hábitat es apta para sus usuarios debido al funcionamiento de un sistema de soporte vital. Este último corresponde al monitoreo ambiental de la temperatura y humedad, control atmosférico o limpieza del aire, reciclaje de aguas servidas y almacenamiento y distribución de agua potable (NASA, 2019). En la industria aeroespacial el sistema de soporte vital es visible. Cables, tuberías, capas de película para proteger partes sensibles a la radiación, los mecanismos de palancas de las compuertas, y todo lo añadido al armazón principal que pueda estar a la vista. Solo se protegen el cableado y aparatos más delicados detrás de un cascarón que a la vez sirve para darle forma definida a los espacios y adaptarlos al flujo humano. En este caso, lo 
aeroespacial está basado en el manejo práctico de sus partes, especialmente en situaciones de concentración total o emergencias.

Habitar en una atmósfera controlada es conveniente en tiempos y lugares de alta contaminación. La protección acústica, junto al sistema de soporte vital, garantizan el bienestar de sus usuarios y, por ende, la calidad de vida aumenta debido al confort logrado. Los ruidos deben ser eliminados del hábitat humano, y para eso existen materiales y estrategias de planificación que pueden asegurarlo. La ausencia del sonido en el vacío indica que la industria aeroespacial ha tratado el tema principalmente para controlar los decibeles que la indumentaria aeroespacial emite hacia los interiores de sus estructuras (Feireiss \& Najjar, 2018). Este conocimiento debe aprovecharse y optimizarse en arquitectura.

Es necesario e importante para la evolución del ambiente construido conocer los avances de la industria aeroespacial, pues sus resultados pueden ser de beneficio para la humanidad mientras se concretizan la exploración y colonización espaciales. La arquitectura, como ambiente artificial total, es una oportunidad para optimizar las cualidades aeroespaciales y poner a prueba sus resultados para, en cambio, ampliar y revisar la implementación cósmica.

\section{Principios originarios}

El análisis de las estrategias aeroespaciales (autenticidad, policromía, exactitud, responsividad y habitabilidad), promueve que la arquitectura se establezca bajo principios que continuamente ajusten nuevos estilos de vida a ambientes artificiales emergentes. Los principios descubiertos son los siguientes:

- La arquitectura es tangible. Aunque está basada en conceptos, principios y planificaciones, la arquitectura es un objeto concreto que ocupa un sitio específico. Estar conscientes de su tangibilidad permite asegurar que su implementación cumpla con los requisitos medioambientales necesarios que garanticen el confort humano y el mejoramiento del lugar a implantarse.

- La arquitectura es moderada. El tamaño de cada una de sus partes debe ser justo, y debe rechazar el uso excesivo de materia y espacio, ajustando el procesamiento, espesor, la constitución y la superficie útil necesaria de cada elemento

- La arquitectura debe evitar tipologías. Arquitectura es una estructura compuesta por dos sectores, uno polivalente que puede tener cambios de uso en el tiempo sin necesidad de hacer modificaciones a la instalación total, y otro inmutable con maquinarias que garanticen confort. Este último debe depender del tamaño vertical de la estructura y la superficie útil derivada del flujo máximo de personas planificado, por ejemplo. La ausencia de tipologías libera a la arquitectura de costumbres culturales efímeras que podrían limitar la vida útil de la misma. Planificar sin requisitos tipológicos promueve la reutilización de arquitecturas, y consigue disminuir la producción de nueva materia prima cada vez que necesite ser rehabilitada.

- La arquitectura exige eficiencia. La eficiencia es una ambición humana que puede evolucionar poniéndola a prueba en estructuras habitables. Las ciencias contemporáneas han llegado a un alto nivel de perfección que controla la existencia de cada objeto desde su planificación y fabricación, hasta su uso y desecho. La arquitectura aprovecha los avances de la computación para optimizar la manufactura de objetos y para garantizar el funcionamiento infalible de sus partes una vez esté construido. La claridad y objetividad de pensamiento también es parte de la eficiencia que persigue toda arquitectura, pues es imprescindible eliminar la oscuridad conceptual que pudiera entorpecer el desarrollo de hábitats futuros; es decir, la eficiencia no es solo material, también debe ser intelectual. 
- La arquitectura tiene vida útil. Las partes de toda estructura artificial son degradadas por accidentes, razones climáticas, uso y contaminación humanos. La arquitectura es una alternativa para demostrar los avances que la ciencia de materiales provee, usando las lecciones de los de última generación, consiguiendo la sección mínima/necesaria de cada pieza y aplicando capas inteligentes que protejan las superficies externas de manchas y bacterias. Se recomienda el uso de materiales con cualidades plásticas (moldeables), longevo, ambientalmente validado, que sea estructura y cierre simultáneamente; con refuerzos internos para contrarrestar fuerzas cortantes y de tensión y con posibilidad de perfeccionar su fórmula para alcanzar una ligereza que facilite su manipulación, ensamblaje y desecho. Es necesario asignar fecha de expiración a aquellos elementos reemplazables para precisar la manufactura, fabricación de los mismos y su ulterior reciclaje.

- La arquitectura es adaptable. La arquitectura no solo debe estar hecha de componentes longevos, también debe ser expansible para extender su vida útil. La planificación de la misma debe asegurar que la expansión no comprometa la estructura que ha sido terminada, es decir, que debe obedecer a un patrón general donde cada elemento garantice la eficiencia de la estructura total. La adaptación implica que está formada por partes prefabricadas, aunque existe la posibilidad de hallar nuevas en el proceso. Para ser adaptable, sus componentes deben ser ensamblables, exigiendo que cada elemento forme parte de un sistema total, ajustando sus formas y tamaños, minimizando la variedad de las partes y utilizando las ventajas de la estandarización y personalización heredadas del siglo pasado

- La arquitectura es una totalidad. La arquitectura debe entenderse como una totalidad, ya que todas sus partes trabajan en unísono para garantizar eficiencia, longevidad y ser adaptable, aprovechando la ventaja de lo artificial, es decir, el beneficio de lo planificado y lo controlado. A diferencia de la costumbre clásica concentrada en la apariencia externa, la arquitectura es una totalidad de infraestructura y caparazón, y como producto de una totalidad conceptual, insiste en la síntesis, objetividad y claridad de pensamiento.

La arquitectura es un todo coherente con límites precisos que delimitan y declaran cuáles elementos forman parte o no del mismo. La misma tiene zonas de amortiguamiento entre lo artificial y lo natural, siendo este último una influencia constante sobre todo el ambiente, y contiene flujo de actividades que eventualmente dejan desechos físicos. Igualmente, la arquitectura exige que su imagen final responda a la infinita variedad de visiones humanas y cibernéticas, apoyándose en componentes intangibles (informadores) y componentes tangibles (formadores).

\section{Componentes intangibles: informadores}

Los informadores son la base intelectual y visual de la arquitectura. Su función es estructurar las posibilidades de un ambiente artificial que pueda llegar a un resultado preciso y sensible. Los informadores son los siguientes:

- Reporte Contextual. El Reporte Contextual indica la localización precisa de cada elemento del sitio, describiendo y almacenando las características físicas e invisibles del lugar. En arquitectura se habla de "contexto" acompañado de data, pues este último es un ecosistema digital cuantitativo y cualitativo. Por ejemplo, un esquema en nube de puntos es una representación cercana a un contexto vivo, pues es un cúmulo de puntos sensibles a acciones digitales tridimensionalmente. Este tipo de data puede concluir 
en un reporte que será la guía de cómo debe abordarse el lugar, evitando modificaciones drásticas que conviertan el sitio en un punto inoperante en el futuro. El Reporte Contextual debe indicar cómo mantener intactos la mayoría de los objetos encontrados en la superficie, en el espacio aéreo correspondiente y en el subsuelo, sugiriendo que en el futuro la arquitectura continúe la tradición de elevarse del suelo para salvaguardar el ecosistema donde se implemente.

- Programa. El programa es la respuesta adecuada a la data levantada y asignada en el Reporte Contextual. La arquitectura debe seguir las recomendaciones obtenidas del reporte para deducir el tipo óptimo de ocupación para ese lugar específico; se debe evitar imponer un uso predeterminado, así como responder a una tipología específica. El programa se desarrolla en las tres coordenadas XYZ, incluyendo las oblicuas que pueden maximizar la comunicación cruzada entre las mismas. El programa se puede alternar durante el horario de uso de la estructura por ser un ambiente artificial con actividad continua aún en las horas de aparente pausa, y puede establecer usos en vez de funciones, pues los usos corresponden al flujo y cantidad de usuarios; no a actividades específicas que resultan eventualmente pasajeras.

- Argumentos. El Reporte Contextual y el programa deben formular argumentos que planteen escenarios de habitabilidad y diseño precisos. Los argumentos pueden producir resultados originales, pues no están basados primariamente en modelos (analogías) o experiencia (inducción). Los argumentos son autoevidentes, y son la base de un razonamiento abstracto para llegar a configuraciones únicas. Los argumentos pueden declarar la arquitectura como un hecho para ser razonado, y pueden promover exactitud para comunicar complejidades abstractas que surjan en la formulación de nuevas visiones arquitectónicas (Kline, 1967).
- Diseño. El diseño es la respuesta a los argumentos, y debe ser única a pesar de haberse revisado en múltiples versiones. El mismo se puede planificar a través de la computación, permitiendo verificar la exactitud de la planificación, fabricación y posición de cada elemento en el conjunto. Debe además, validar el Reporte Contextual, el programa y los argumentos; tener dimensiones para identificarse con lo humano, tamaños que vayan más allá del cálculo y respondan a la cultura del proyectista. El diseño es donde se incluyen las estrategias transmitidas por la industria aeroespacial (autenticidad, policromía, exactitud, responsividad y habitabilidad) o de otro campo que pueda evolucionar la arquitectura y liberarla de sus dogmas.

\section{Componentes tangibles: \\ formadores}

Los formadores son elementos tangibles que le dan característica evidente a la arquitectura. Cada uno y sus partes secundarias deben seguir la misma lógica de todo el sistema, logrando que su constitución física, operativa e instalación respondan a los principios originarios y a los informadores. Los elementos formadores son los siguientes: pilotes, instalaciones, armazón, maquinarias y caparazón.

- Pilotes. Los pilotes son elementos subterráneos de anclaje. Es lógico tener el menor impacto posible sobre el terreno natural (como lo sugiere el Reporte Contextual), y para lograrlo sus fundaciones puntuales deben ser las mínimas que el diseño indique, permitiendo levantar todo el conjunto del suelo original. Igualmente, se persigue salvaguardar el subsuelo de cualquier contaminación y modificación drástica a su constitución, y se debe insistir en mantener lo autóctono del contexto a escala humana y aérea, pues es el primer punto de encuentro entre lo natural y lo artificial. 
- Instalaciones. El suelo natural debe ser ocupado por instalaciones artísticas que faciliten usos complementarios. Las instalaciones deben, al igual que el resto de la estructura, estar levantados moderadamente sobre la superficie para permitir que el flujo de vida natural continúe. Las instalaciones pueden regenerar el suelo y la vegetación, servir de contenedores de agua y ayudar a limpiar el aire circundante usando sistemas indicados en los argumentos.

- Armazón. El armazón es la estructura principal, y como tal, es la continuación estratégica de las pilotes. Los materiales y especificaciones de estabilidad y comportamiento responden a los cálculos y advertencias indicadas en los argumentos y el diseño. El armazón debe ser modular, nunca producido in situ, y debe estar preparado para expandirse o desarmarse eventualmente causando el menor impacto posible a las condiciones naturales del lugar.

- Maquinarias. Las maquinarias son la indumentaria de habitabilidad, climática, energética y circulación que mantienen la arquitectura en operación. Son entidades autocontenidas, y pueden venir prefabricadas, completamente listas para instalarse en las cámaras de equipamiento. La reducción del tamaño de los objetos de uso cotidiano y de las máquinas podría plantear dificultades para la precisión, pero eso se resuelve durante el diseño, planificando las cámaras de equipamiento para usos futuros de antemano.

- Caparazón. El caparazón está compuesto de paneles preindustrializados, y determina la apariencia externa e interna de la arquitectura. El armazón puede permanecer casi intacto a lo largo de la vida útil, mientras que el caparazón puede cambiar de acuerdo a los requerimientos de nuevos estilos de vida. Esto instaura un sistema de remplazo de piezas, y elimina la necesidad de demoler elementos cada vez que se readapte el espacio.
Las costumbres humanas son cambiantes y exigen nuevas apariencias de ambiente, pero no necesariamente de la infraestructura (lo más costoso). Esto puede convertir a la arquitectura en un sistema costo-eficiente, que por cambiar raras veces sus partes fundamentales, contribuye con la estabilidad del ecosistema donde se ubique.

\section{Hacia una libertad intelectual total}

Toda transferencia de conocimiento es parcial. La arquitectura puede aprovechar los procesos de planificación, control atmosférico, manejo del agua, confort ambiental y la lógica de nuevos materiales de construcción heredados del mundo aeroespacial, aunque no debería aplicarlos literalmente, sino abstraerlos y transformarlos en estrategias de diseño que ayuden a la formulación de nuevos hábitats como se ha introducido en el presente artículo.

La arquitectura debe seguir evolucionando hacia una alternativa inteligente que reforme el entorno construido aprovechando el conocimiento de profesiones foráneas como la industria aeroespacial y campos compatibles. Paralelamente, debe evitar los dogmas que esas profesiones imponen, creando los propios para abrir paso a estructuras que respondan a las realidades cambiantes de la humanidad y sus ecosistemas. Eso es posible si se aleja de la clasificación por estilos o del enfoque técnico como única salida. Los estilos han sido importantes para la historia porque ayudan a argumentar usando conceptos familiares, y las discusiones técnicas son útiles en procesos constructivos, pero lo fundamental está en la intersección de conocimientos inclinados a optimizar el hábitat humano, algo que la industria aeroespacial enfatiza.

La interdisciplinariedad vista a través de las estrategias, los principios y los componentes enunciados fomenta un equipo híbrido humano/ artificial. Esto podría inspirar una arquitectura que se enseñe bajo modelos educativos evolucionados, con metodologías que desarrollen el 
conocimiento holístico que el medioambiente deteriorado exige. Igualmente, es importante revisar las bases de datos de las academias antes de continuar formulando nuevas alternativas profesionales, pues hoy día existen descubrimientos inéditos, producidos desde el pasado milenio en las universidades, que podrían solucionar los problemas que se están multiplicando en el presente siglo. Esta interdisciplinariedad híbrida puede optimizar el tiempo de implementación de nuevas ideas.

La función democrática de la arquitectura es implementar nuevas visiones basadas en dinámicas interdisciplinarias, y esa es una libertad que necesita debatirse dentro de los ambientes concretos que surgen en la práctica profesional. Igualmente, esa libertad interdisciplinaria, apoyada en la investigación y planificación, establece que la arquitectura pertenece a un complejo mayor (la ciudad) el cual exige que todos los campos involucrados salvaguarden los limitados recursos que posee el planeta. Finalmente, esa función promueve que ningún campo sea dominante, sino que todos se enfoquen hacia lo que es adecuado, hacia un devenir conveniente para todos, hacia una libertad intelectual total.

\section{Referencias}

Asimov, I. (1974). Our world in space. Greenwich, Conn.: New York Graphic Society, Ltd.

Casey, R. \& McWilliams, C. (2010). Form + code. Princeton Architectural Press.

Castillo Molina, JR. (2019). Innovación y transferencia: La implementación del hallazgo. AULA Revista de Humanidades y Ciencias Sociales, 64 (4), 46-54 https: \\doi. org $\backslash 10.33413 \backslash$ aulahcs.2019.64i4.102

Cole, E. (2006). La gramática de la arquitectura. Lisma Ediciones, S.L.

Dunn, N. (2012). Proyecto y construcción digital en arquitectura. Art Blume, S.L.

Feiress, L. \& Najjar, M. (2018). Planetary echoes. Spector Books.

Garner, P. (1996). Sixties design. Taschen GmbH.

Kline, M. (1967). Mathematics for the nonmathematician. General Publishing Company, Lrd.

McCarter, R. (1987). Building machines. Princeton Architectural Press.

Magomedov, S. (2015). Georgii Krutikov: the flying city and beyond. Editorial Tenov.

Ota, K. (2011). Project Japan: Metabolism talks... TASCHEN.

Oxman, R. \& Oxman, R. (2010). The new structuralism: design, engineering and architectural technologies. Architectural Design, p.14-23. https: \\oi.org \10.1002\ad.1101

Rams, D. (2014). Less but better. Jo Klatt Design + Design Verlag.

Sasaki, M. (2005). Flux structure. Toto Publishings.

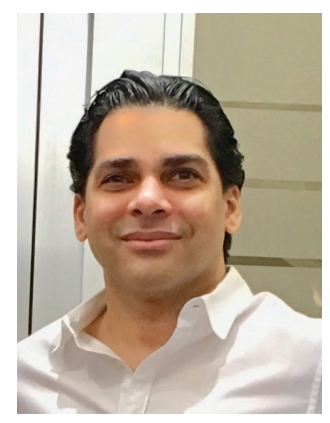

\section{Juan R. Castillo}

Arquitecto egresado de Arquitectura y Urbanismo (UNPHU, 1994), obtuvo Doctorado en Hiroshima University (Japón, 2002), jurado de maestría en Columbia University (USA, 2010), es profesor titular en la Universidad Iberoamericana (UNIBE), coordinador de Innovación Arquitectónica (UNPHU) y editor jefe de la revista UMBRIEL de la UNPHU. Ha publicado en Journal of Architecture, Planning and Environmental Engineering (AIJ, Tokyo), Misawa Bauhaus Collection (Tokyo), Portal Docente UNIBE y Aula, Revista de Humanidades y Ciencias Sociales. Asociado a Shearly Investments, Inc., diseñó proyectos turísticos, habitacionales e industriales. Miembro del Architectural Institute of Japan (AIJ, Tokyo), CODIA y SARD (Santo Domingo), jurado del concurso ADOACERO, y practica desde su firma FUTURA, Arquitectura Emergente SRL. 